\title{
Usability Evaluation of Packaging Design Based on Eye Movement Experiment
}

\author{
Weijun Zhao ${ }^{1,}$, Linyan Zheng ${ }^{1, b}$ and Tian Wang ${ }^{1, c}$ \\ ${ }^{1}$ School of Packaging Design \& Art, Hunan University of Technology, Zhuzhou, Hunan, 412007, \\ China \\ a zwj706@163.com, b569030738@qq.com, c495936371@qq.com
}

Keywords: Usability evaluation, Packaging design, Eye movement experiment

\begin{abstract}
With the fast development of packaging design, the usability evaluation has been paid much attention to. On the other hand, with the popularity of the eye movement experiment, the evaluation method based on experimental data has been introduced into the usability evaluation system. This paper, taking the packaging of MG mask as the target, evaluates the design usability by eye movement experiment in terms of packaging-opening and shelf display. The results indicate that customers accepted the packaging design. The opening of the first-layer packaging was time-consuming, however, and the inner packaging needed to improve. Finally, some suggestions are presented for the betterment of the packaging design.
\end{abstract}

\section{Introduction}

As the rapid growth of packaging industry, packaging design has been paid much attention to. Thus, packaging design evaluation has been discussed more ever than before, and design usability evaluation is an important problem now. The advent of eye-tracking equipment has led in recent years to a growth of researches into the deployment of visual attention and eye movements in design evaluation, which has presented more reasonable and scientific measurement on packaging design. However, many researchers have focused on the applications of eye movement to readings, information processing[1-2], perception in art[3-4] and real-world scene perception[5-6], insufficient studies are related to usability evaluation.

Generally speaking, the study on theories and methods of design usability is still at an early stage in China[7]. Usability evaluation is closely related with consumers' demands and designers' ideas. Therefore, new evaluation methods will be helpful to the development of packaging design.

\section{Packaging Design Usability and Eye Movement Evaluation}

Definition. By eye tracking equipment, some eye movements can be observed so that designers can know which element can attract the customers most and which can be improved.

Usability evaluation of packaging design based on eye movement means that people evaluate such elements as the structure, the shape, the colors and the characters of a package by eye tracking technique according to usability principles to determine the inherent functions that a packaging design should meet, thus measuring the values of the package in terms of product marketing, brand establishment, environmental protection and its cultural and social attributes.

Evaluation Index Selection. According to the life cycle of a package, combined with its shape, materials and functions, the evaluation indices are selected to decide whether the usability principles are followed. The comprehensive index system includes such elementary functions as protection, airproof, transportation, convenience, adaptability, ecology and humanity.

The packaging usability indices include the following three second-level indices: functionality, ease of use and efficiency. Functionality consists of physical and mental indices. And, ease of use and efficiency are used to evaluate the application and economic values of the targeted packaging design.

\section{Eye Movement Experimental Design of Packaging Usability Evaluation}


Taking the packages of MG Eye Mask as the target, we first used market survey and questionnaires to analyze the design usability. And then, we evaluated the packaging-opening and shelf display by eye movement, respectively. Next, we analyzed the advantages and disadvantages of the targeted packaging design based on experimental data to present suggestions for design improvements.

Package-opening Experiment. In package-opening experiment, 20 people were selected and then were equally divided into 4 groups to open the packages. The durations used for opening the first-layer of the package were 15, 16, 12 and 6 seconds, respectively. The durations for opening the second layer were 11, 1, 20 and 7 seconds, respectively. So the average opening time was 10 seconds. The durations for taking out the products after opening were 2, 5, 5, and 6 seconds, respectively.

The subjects of the four groups didn't cause serious damage to the packages while opening the first-layer of the package (as shown in Fig.1), whereas, it was a little difficult to open the label sealing area. As for the opening of the second-layer of the package (as shown in Fig.1), the subjects thought the packaging surface was so smooth that they had to keep their hands dry to open the packaging. Finally, when opening the inner packaging, the subjects found that some essence lotion spilled out.
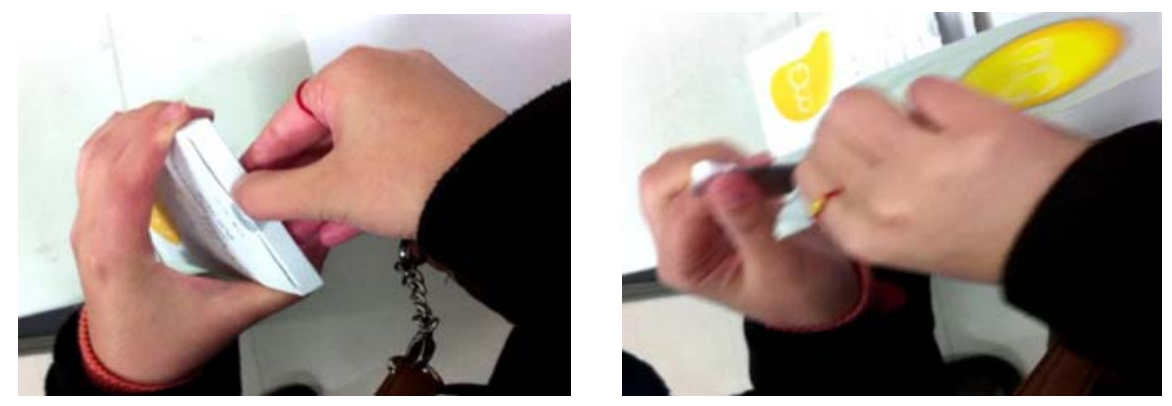

Fig.1 Opening of the first-layer and inner packaging of MG eye mask

After the opening experiment, an investigation was held. The results showed that about 10 percent of the subjects had little purchasing desire at the first sight of the packaging, about 65 percent had certain desire to buy the mask, and 25 percent had strong desire to buy the mask. Totally speaking, customers' first impression of MG mask packaging design was fairly positive. As for packaging-opening evaluation, 70 percent subjects thought it was easy to open the packaging, 15 percent thought the packaging was of great convenience, and none of the interviewees was unsatisfactory. More than 40 percent well accepted MG packaging design.

Eye Movement Experirments on Shelf Display. Generally speaking, the LOGO on MG packaging is very concise, easy to distinguish, and the information printed is very clear. The packaging design is attractive and reflects humanity and personality.
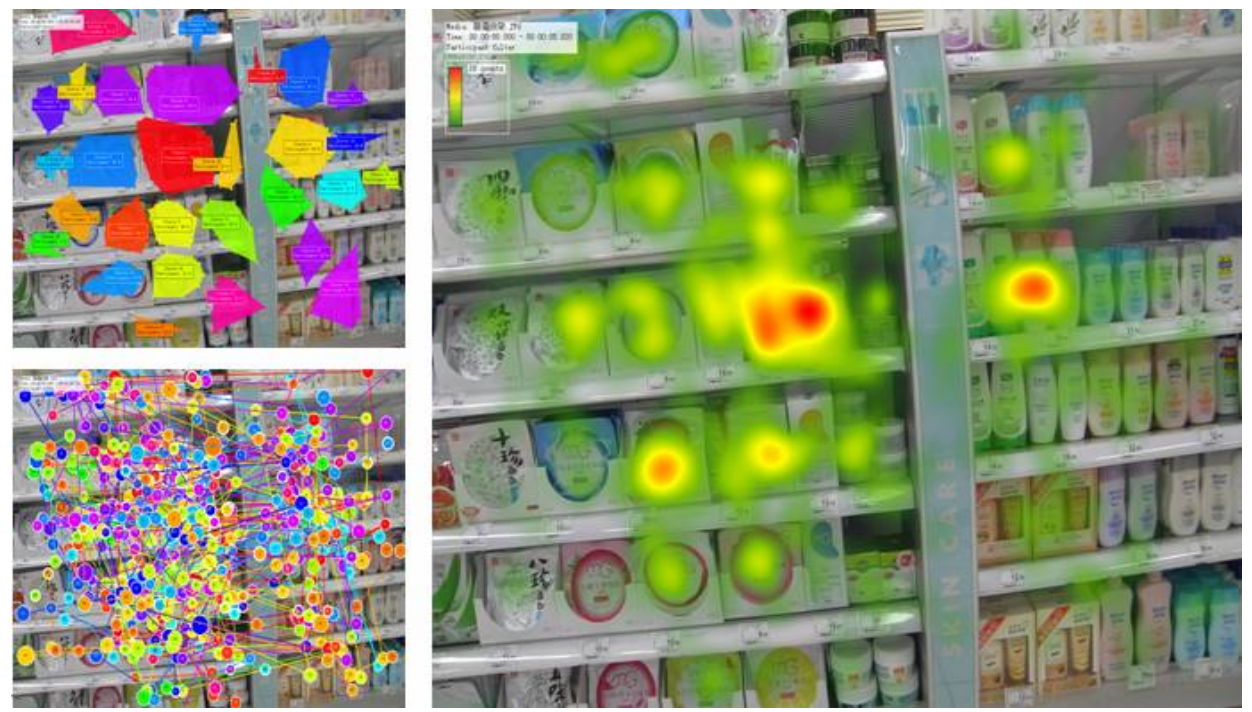

Fig.2 Heat Maps of Eye movement of MG mask packaging 
By eye movement tracker, we made experiments on the shelf display of MG masks, and obtained the heat map(as shown in Fig.2) and Area of Intersts(AOI)(as shown in Fig.3).

As seen in the heat maps of the shelf display, MG mask packaging could attract customers' attention and made them fix on it for a fairly long time. Thus, the packaging was well designed. Before purchasing decisions, the packaging design could deliver effective information and made them focus on the brand and the product. This can explain to some degree that MG masks have a pretty high market share.

Then we discussed the results based on the following two aspects: time to first fixation and total fixation duration.

We divided MG mask packaging into two AOIs(as shown in Fig.3). Purple means the area of the packaging shape(AOI1), and blue means the area of characters of the packaging(AOI2). The data indicated that the average time to first fixation on AOI1 was 0.53 seconds and that on AOI2 was 0.46 seconds. But the total fixation duration was more than 23 seconds. Furthermore, the experimental data reflected that the time to fixation on characters printed was longer than that on the shape.

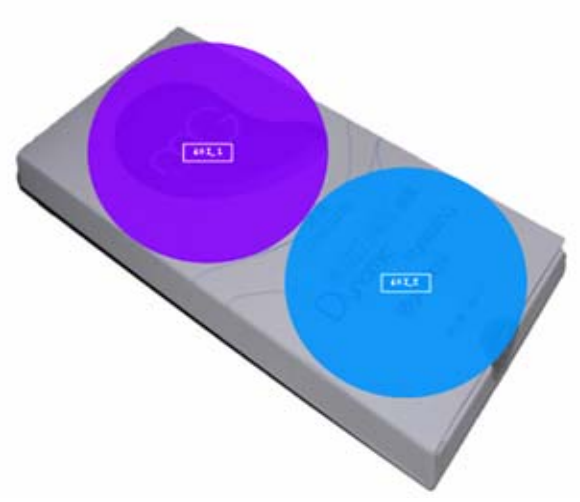

Fig. 3 AOIs of MG mask packaging

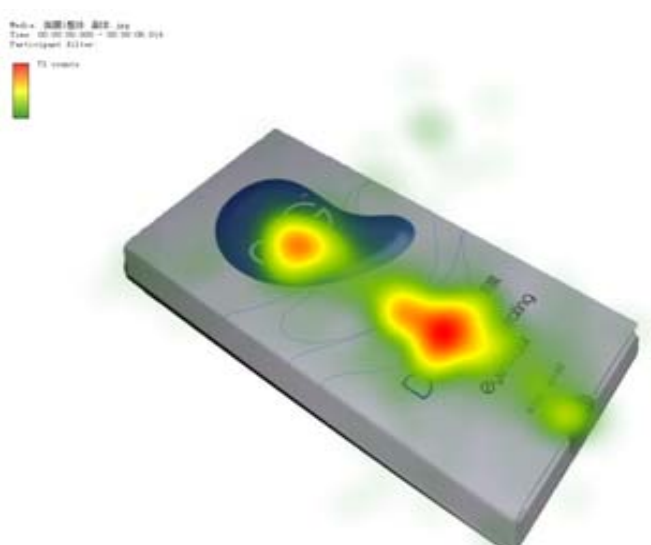

Fig.4 Heat map of the outer packaging

According to the heat map( as shown in Fig.4), the eye movement of the subjects focused on product patterns and characters, and they fixed longer on the characters. The result, from another perspective, pointed that customers may spend more time on product information on the packaging before purchasing decisions. However, characters on MG packaging are unequal in thickness and are distinguished in colors, which results in less visual layers.

\section{Discussion}

By eye movement experiment, we can learn customers’ opinions on the targeted packaging design. The key to a good packaging design should manifest itself in usability such as packaging materials, shapes, colors and so on. Then, according to the experimental data, we will discuss the targeted packaging design in terms of functionality, ease of use and efficiency to present some problems in packaging design. And furthermore, some suggestions will be put forward for design improvements. Evaluation of Packaging Functionality. The packaging materials of MG products are selected based on the physical shape and chemical attributes of the products packed inside, which meets the needs of protection in transportation and usage. The quality of the materials and compatibility of the materials and the products both measure up to the related standards. The outer part of the packaging accords with printing standards.

In terms of physical index, MG mask packaging has achieved desired functions, as the application of PE materials can prevent the moisture and essence lotion from spilling out and evaporating. The attributes of waterproof and airproof are satisfactory. But the sealing is not very good, heat sealing technique may be considered to secure a firm sealing. 
In terms of mental index, the dyeability of PE composite material used on MG mask packaging is really excellent, and the trueness and the brightness of the colors are well reflected.

Evaluation of Ease of Use. From the above experiment, we obtained that the packaging design of MG masks have the following characteristics: (1)time used to open the first-layer packaging is a little longer; (2) the inner packaging is too moist and the sealing is not firm. These disadvantages may have negative impacts on further using and purchasing.

On the other hand, the application of carton packaging has many unique properties, and is tailored to the psychological demands of Chinese customers. In China, paper is made from wood which means the essence of life and the respect for natural life.

Evaluation of Packaging Efficiency. The inner product is well-packed by bags made from PE composite materials. This kind of material is of excellent airproof, sun-block and high biodegradability, which agrees with the trend in modern packaging design.

\section{Conclusions}

Recently, with the popularity of eye tracker and the establishment of eye movement laboratories, eye movement experiments have been used to evaluate usability of packaging design, which makes it possible to analyze and evaluate packaging design by quantitive and more reliable methods, and promote packaging designed for customers' needs.

This paper, taking the packaging of MG mask as the target, evaluates the usability of packaging design by eye movement experiments. Totally speaking, customers accepted the packaging design, the opening of the first-layer was time-consuming, however, and the inner packaging needed to improve. Finally, some suggestions are presented for the betterment of the packaging design. The usability design evaluation based on eye movement not only can improve objectivity and scientificity of the packaging design evaluation, but also can open a new direction for the development of packaging design.

\section{Acknowledgements}

This work is financially supported by National Social Science Foundation (12BG053) and by the project of Provincial Planning Office of Philosophy and Social Science of Hunan (12YBB076).

\section{References}

[1] K. Rayner, Eye movement in reading and information processing, Psychological Bulletin. 124 (1998) 373-422.

[2] K. Rayner, Eye movement and attention during reading, scene perception, and visual search, Quarterly Journal of Experimental Psychology. 62 (2009) 1457-1506.

[3] G.R. Loftus, Eye fixation and recognition memory for pictures, Cognitive Psychology. 3(1972)618-660.

[4] G.Underwood, Eye Guidance in Reading and Scene Perception, first ed., Elsevier Science Ltd, Amsterdam, 1998.

[5] J.M. Henderson, Introduction to real-world scene perception, Visual Cognition.12(2005)849-851.

[6] Roger R.G. Van Gomple, Martin H Fisher, Wayne S Murray and Robin L.Hill, Eye Movements: a Window on Mind and Brain, first ed., Elsevier Science Ltd, Amsterdam, 2007.

[7]L.Z. Ge, L.Wan, Review of Packaging Usability Research, Packaging Engineering. 31(2010)132-136.(in Chinese) 